\title{
APPROXIMATION ERRORS IN TRUNCATED DIMENSIONAL DECOMPOSITIONS
}

\begin{abstract}
SHARIF RAHMAN
ABSTRACT. The main theme of this paper is error analysis for approximations derived from two variants of dimensional decomposition of a multivariate function: the referential dimensional decomposition (RDD) and analysisof-variance dimensional decomposition (ADD). New formulae are presented for the lower and upper bounds of the expected errors committed by bivariately and arbitrarily truncated RDD approximations when the reference point is selected randomly, thereby facilitating a means for weighing RDD against ADD approximations. The formulae reveal that the expected error from the $S$-variate RDD approximation of a function of $N$ variables, where $0 \leq S<N<\infty$, is at least $2^{S+1}$ times greater than the error from the $S$-variate ADD approximation. Consequently, ADD approximations are exceedingly more precise than RDD approximations. The analysis also finds the RDD approximation to be sub-optimal for an arbitrarily selected reference point, whereas the ADD approximation always results in minimum error. Therefore, the RDD approximation should be used with caution.
\end{abstract}

\section{INTRODUCTION}

Uncertainty quantification of complex systems mandates stochastic computations of a multivariate output function $y$ that depends on $\mathbf{X}:=\left(X_{1}, \cdots, X_{N}\right)$, a high-dimensional random input with a positive integer $N$. For practical applications, encountering hundreds of variables or more is not uncommon, where a function of interest, defined algorithmically via numerical solution of algebraic, differential, or integral equations, is all too often expensive to evaluate. Therefore, there is a need to develop low-dimensional approximations of $y$ by seeking to exploit the hidden structure potentially lurking underneath a function decomposition.

The dimensional decomposition of

$$
y(\mathbf{X})=\sum_{u \subseteq\{1, \cdots, N\}} y_{u}\left(\mathbf{X}_{u}\right)
$$

can be viewed as a finite, hierarchical expansion in terms of its input variables with increasing dimensions, where $u \subseteq\{1, \cdots, N\}$ is a subset with the complementary set $-u=\{1, \cdots, N\} \backslash u$ and cardinality $0 \leq|u| \leq N$, and $y_{u}$ is a $|u|$ variate component function describing a constant or the cooperative influence of $\mathbf{X}_{u}=\left(X_{i_{1}}, \cdots, X_{i_{|u|}}\right), 1 \leq i_{1}<\cdots<i_{|u|} \leq N$, a subvector of $\mathbf{X}$, on $y$ when $|u|=0$ or $|u|>0$. The summation in (1.1) comprises $2^{N}$ terms, with each term depending on a group of variables indexed by a particular subset of $\{1, \cdots, N\}$, including the

Received by the editor May 30, 2011 and, in revised form, June 7, 2012.

2010 Mathematics Subject Classification. Primary 41A63, 41A99, 26B99, 65G99, 65C60.

Key words and phrases. Uncertainty quantification, ANOVA, HDMR, ADD, RDD.

The author was supported in part by NSF Grant \#CMMI-0969044 and \#CMMI-1130147. 
empty set $\emptyset$. This decomposition, first presented by Hoeffding [1] in relation to his seminal work on $U$-statistics, has been studied by many other researchers [2]: Sobol [3] used it for quadrature and analysis of variance (ANOVA) 44; Efron and Stein [5] applied it to prove their famous lemma on jackknife variances; Owen [6] presented a continuous space version of the nested ANOVA; Hickernell [7] developed a reproducing kernel Hilbert space version; and Rabitz and Alis 8 made further refinements, referring to it as high-dimensional model representation (HDMR). More recently, the author's group formulated this decomposition from the perspective of Taylor series expansion, solving a number of stochastic-mechanics problems [9 11].

In a practical setting, the multivariate function $y$, fortunately, has an effective dimension 12 much lower than $N$, meaning that $y$ can be effectively approximated by a sum of lower-dimensional component functions $y_{u},|u| \ll N$. Given an integer $0 \leq S<N$, the truncated dimensional decomposition

$$
\hat{y}_{S}(\mathbf{X})=\sum_{\substack{u \subseteq\{1, \cdots, N\} \\ 0 \leq|u| \leq S}} y_{u}\left(\mathbf{X}_{u}\right)
$$

then represents a general $S$-variate approximation of $y(\mathbf{X})$, which for $S>0$ includes cooperative effects of at most $S$ input variables $X_{i_{1}}, \cdots, X_{i_{S}}, 1 \leq i_{1}<\cdots<i_{S} \leq$ $N$, on $y$. However, for (1.2) to be useful, one must ask the fundamental question: what is the approximation error committed by $\hat{y}_{S}(\mathbf{X})$ for a given $0 \leq S<N$ ? The answer to this question, however, is neither simple nor unique, because there are multiple ways to construct the component functions $y_{u}, 0 \leq|u| \leq S$, spawning approximations of distinct qualities. Indeed, there exist two important variants of the decomposition: (1) referential dimensional decomposition (RDD) and (2) ANOVA dimensional decomposition (ADD), both representing sums of lower-dimensional component functions of $y(\mathbf{X})$. While ADD has desirable orthogonal properties, the ANOVA component functions are difficult to compute due to the high-dimensional integrals involved. In contrast, the RDD lacks orthogonal features with respect to the probability measure of $\mathbf{X}$, but its component functions are much easier to obtain. For RDD, an additional question arises regarding the reference point, which, if improperly selected, can mar the approximation. Existing error analysis, limited to the univariate truncation of $y(\mathbf{X})$, reveals that the expected error from the RDD approximation is at least four times larger than the error from the ADD approximation [13. Although useful to some extent, such a result alone is not adequate when evaluating multivariate functions requiring higher-variate interactions of input 9 11]. No error estimates exist yet in the current literature, even for a bivariate approximation. Therefore, a more general error analysis pertaining to a general $S$-variate approximation of a multivariate function should provide muchneeded insight into the mathematical underpinnings of dimensional decomposition.

The purpose of this paper is twofold. Firstly, a brief exposition of ADD and RDD is given in Section 2, including clarifications of parallel developments and synonyms used by various researchers. The error analysis pertaining to the ADD approximation is described in Section 3. Secondly, a direct form of the RDD approximation, previously developed by the author's group, is tapped for providing a vital link to subsequent error analysis. Section 4 introduces new formulae for the lower and upper bounds of the expected errors from the bivariate and general $S$-variate RDD approximations. These error bounds, so far available only for the 
univariate approximation, are used to clarify why ADD approximations are exceedingly more precise than RDD approximations. There are seven new results stated or proved in this paper: Proposition 3.1, Theorems 4.6, 4.12, and Corollaries 4.8, 4.13, 4.15, 4.16. Proofs of other results can be obtained from the references cited, including a longer version of this paper (http://www.engineering.uiowa.edu/ rahman/moc_longpaper.pdf). Conclusions are drawn in Section 5.

\section{Dimensional Decomposition}

Let $\mathbb{N}, \mathbb{N}_{0}, \mathbb{Z}, \mathbb{R}$, and $\mathbb{R}_{0}^{+}$represent the sets of positive integer (natural), nonnegative integer, integer, real, and non-negative real numbers, respectively. For $k \in$ $\mathbb{N}$, denote by $\mathbb{R}^{k}$ the $k$-dimensional Euclidean space and by $\mathbb{N}_{0}^{k}$ the $k$-dimensional multi-index space. These standard notations will be used throughout the paper.

Let $(\Omega, \mathcal{F}, P)$ be a complete probability space, where $\Omega$ is a sample space, $\mathcal{F}$ is a $\sigma$-field on $\Omega$, and $P: \mathcal{F} \rightarrow[0,1]$ is a probability measure. With $\mathcal{B}^{N}$ representing the Borel $\sigma$-field on $\mathbb{R}^{N}$, consider an $\mathbb{R}^{N}$-valued independent random vector $\mathbf{X}:=$ $\left(X_{1}, \cdots, X_{N}\right):(\Omega, \mathcal{F}) \rightarrow\left(\mathbb{R}^{N}, \mathcal{B}^{N}\right)$, which describes the statistical uncertainties in all system and input parameters of a given stochastic problem. The probability law of $\mathbf{X}$ is completely defined by its joint probability density function $f_{\mathbf{X}}: \mathbb{R}^{N} \rightarrow$ $\mathbb{R}_{0}^{+}$. Assuming independent coordinates of $\mathbf{X}$, its joint probability density $f_{\mathbf{X}}(\mathbf{x})=$ $\prod_{i=1}^{i=N} f_{i}\left(x_{i}\right)$ can be expressed by a product of marginal probability density functions $f_{i}$ of $X_{i}, i=1, \cdots, N$, defined on the probability triple $\left(\Omega_{i}, \mathcal{F}_{i}, P_{i}\right)$ with a bounded or an unbounded support on $\mathbb{R}$.

Consider a non-negative, multiplicative, otherwise general, weight function $w$ : $\mathbb{R}^{N} \rightarrow \mathbb{R}_{0}^{+}$, satisfying $w(\mathbf{x})=\prod_{i=1}^{N} w_{i}\left(x_{i}\right)$ with the marginal weight functions $w_{i}: \mathbb{R} \rightarrow \mathbb{R}_{0}^{+}, i=1, \cdots, N$. For $u \subseteq\{1, \cdots, N\}$, let $w_{-u}\left(\mathbf{x}_{-u}\right):=\prod_{i=1, i \notin u}^{N} w_{i}\left(x_{i}\right)$ define the joint weight function associated with $-u$. Without loss of generality, assume that the weight functions have been normalized to integrate to $\int_{\mathbb{R}} w_{i}\left(x_{i}\right) d x_{i}=$ $\int_{\mathbb{R}^{N-|u|}} w_{-u}\left(\mathbf{x}_{-u}\right) d \mathbf{x}_{-u}=\int_{\mathbb{R}^{N}} w(\mathbf{x}) d \mathbf{x}=1$. Let $y(\mathbf{X}):=y\left(X_{1}, \cdots, X_{N}\right)$, a realvalued, measurable transformation on $(\Omega, \mathcal{F})$, define a stochastic response of interest and $\mathcal{L}_{2}(\Omega, \mathcal{F}, P)$ represent a Hilbert space of square-integrable functions $y$ with respect to the induced generic measure $w(\mathbf{x}) d \mathbf{x}$ supported on $\mathbb{R}^{N}$. The representation in (1.1) is called dimensional decomposition if the component functions $y_{u}$, $u \subseteq\{1, \cdots, N\}$, are uniquely determined from the requirements

$$
\int_{\mathbb{R}} y_{u}\left(\mathbf{x}_{u}\right) w_{i}\left(x_{i}\right) d x_{i}=0 \text { for } i \in u \text {. }
$$

Indeed, integrating (1.1) with respect to the measure $w_{-u}\left(\mathbf{x}_{-u}\right) d \mathbf{x}_{-u}$, that is, over all variables except $\mathbf{x}_{u}$, and using (2.1) yields a recursive form

$$
\begin{aligned}
y(\mathbf{X}) & =\sum_{u \subseteq\{1, \cdots, N\}} y_{u}\left(\mathbf{X}_{u}\right), \\
y_{\emptyset} & =\int_{\mathbb{R}^{N}} y(\mathbf{x}) w(\mathbf{x}) d \mathbf{x}, \\
y_{u}\left(\mathbf{X}_{u}\right) & =\int_{\mathbb{R}^{N-|u|}} y\left(\mathbf{X}_{u}, \mathbf{x}_{-u}\right) w_{-u}\left(\mathbf{x}_{-u}\right) d \mathbf{x}_{-u}-\sum_{v \subset u} y_{v}\left(\mathbf{X}_{v}\right),
\end{aligned}
$$

of the decomposition with $\left(\mathbf{X}_{u}, \mathbf{x}_{-u}\right)$ denoting an $N$-dimensional vector whose $i$ th component is $X_{i}$ if $i \in u$ and $x_{i}$ if $i \notin u$. When $u=\emptyset$, the sum in the last line of (2.2) vanishes, resulting in the expression of the constant function $y_{\emptyset}$ in the second 
line. When $u=\{1, \cdots, N\}$, the integration in the last line of (2.2) is on the empty set, reproducing (1.1) and hence finding the last function $y_{\{1, \cdots, N\}}$. Indeed, all component functions of $y$ can be obtained by literally interpreting the last line of (2.2). On inversion, (2.2) results in

$$
y(\mathbf{X})=\sum_{u \subseteq\{1, \cdots, N\}} \sum_{v \subseteq u}(-1)^{|u|-|v|} \int_{\mathbb{R}^{N-|v|}} y\left(\mathbf{X}_{v}, \mathbf{x}_{-v}\right) w_{-v}\left(\mathbf{x}_{-v}\right) d \mathbf{x}_{-v},
$$

providing an explicit form of the same decomposition.

It is important to emphasize that the measure involved in expressing the dimensional decomposition in (2.2) or (2.3) may or may not represent the probability measure of $\mathbf{X}$. Indeed, different measures will create distinct yet exact representations of $y$, all exhibiting the same structure of (1.1). There exist two important variants of dimensional decomposition, described as follows.

2.1. ADD. The ADD is generated by selecting the probability measure of $\mathbf{X}$ as the generic measure, that is, $w(\mathbf{x}) d \mathbf{x}=f_{\mathbf{X}}(\mathbf{x}) d \mathbf{x}$ in (2.2) or (2.3), yielding the recursive form

$$
\begin{aligned}
y(\mathbf{X}) & =\sum_{u \subseteq\{1, \cdots, N\}} y_{u, A}\left(\mathbf{X}_{u}\right), \\
y_{\emptyset, A} & =\int_{\mathbb{R}^{N}} y(\mathbf{x}) f_{\mathbf{X}}(\mathbf{x}) d \mathbf{x}, \\
y_{u, A}\left(\mathbf{X}_{u}\right) & =\int_{\mathbb{R}^{N-|u|}} y\left(\mathbf{X}_{u}, \mathbf{x}_{-u}\right) f_{-u}\left(\mathbf{x}_{-u}\right) d \mathbf{x}_{-u}-\sum_{v \subset u} y_{v, A}\left(\mathbf{X}_{v}\right),
\end{aligned}
$$

that is commonly found in the ANOVA literature [4,5, although for the uniform probability measure $d \mathbf{x}$. The explicit version takes the form

$$
y(\mathbf{X})=\sum_{u \subseteq\{1, \cdots, N\}} \sum_{v \subseteq u}(-1)^{|u|-|v|} \int_{\mathbb{R}^{N-|v|}} y\left(\mathbf{X}_{v}, \mathbf{x}_{-v}\right) f_{-v}\left(\mathbf{x}_{-v}\right) d \mathbf{x}_{-v},
$$

where $f_{-v}\left(\mathbf{x}_{-v}\right)=\prod_{i=1, i \notin v}^{N} f_{i}\left(x_{i}\right)$ is the marginal probability density function of $\mathbf{X}_{-v}:=\mathbf{X}_{\{1, \cdots, N\} \backslash v}$. Equations (2.4) and (2.5) can also be derived from other perspectives, including commuting projections on a linear space of real-valued functions invoked by Kuo et al. 14] for the uniform probability measure.

If $\mathbb{E}$ is the expectation operator with respect to the measure $f_{\mathbf{X}}(\mathbf{x}) d \mathbf{x}$, then two important properties of the ADD component functions, inherited from (2.1), are as follows.

Proposition 2.1. The ADD component functions $y_{u, A}, \emptyset \neq u \subseteq\{1, \cdots, N\}$, have zero means, i.e.,

$$
\mathbb{E}\left[y_{u, A}\left(\mathbf{X}_{u}\right)\right]=\int_{\mathbb{R}^{|u|}} y_{u, A}\left(\mathbf{x}_{u}\right) f_{u}\left(\mathbf{x}_{u}\right) d \mathbf{x}_{u}=0 .
$$

Proposition 2.2. Two distinct $A D D$ component functions $y_{u, A}$ and $y_{v, A}$, where $\emptyset \neq u \subseteq\{1, \cdots, N\}, \emptyset \neq v \subseteq\{1, \cdots, N\}$, and $u \neq v$, are orthogonal, i.e., they satisfy the property

$$
\mathbb{E}\left[y_{u, A}\left(\mathbf{X}_{u}\right) y_{v, A}\left(\mathbf{X}_{v}\right)\right]=\int_{\mathbb{R}|u \cup v|} y_{u, A}\left(\mathbf{x}_{u}\right) y_{v, A}\left(\mathbf{x}_{v}\right) f_{u \cup v}\left(\mathbf{x}_{u \cup v}\right) d \mathbf{x}_{u \cup v}=0 .
$$


Traditionally, (2.4) or (2.5) with $X_{j}, j=1, \cdots, N$, following independent, standard uniform distributions, has been identified as the ANOVA decomposition [4; however, the author's recent works [15, 16, reveal no fundamental requirement for a specific probability measure of $\mathbf{X}$, provided that the resultant integrals in (2.4) or (2.5) exist and are finite. In this work, the ADD should be interpreted with respect to an arbitrary but product type probability measure for which it is always endowed with desirable orthogonal properties. However, the ADD component functions are difficult to ascertain, because they require calculation of high-dimensional integrals.

2.2. RDD. Consider a reference point $\mathbf{c}=\left(c_{1}, \cdots, c_{N}\right) \in \mathbb{R}^{N}$ and the associated Dirac measure $\prod_{i=1}^{N} \delta\left(x_{i}-c_{i}\right) d x_{i}$. The RDD is created when $\prod_{i=1}^{N} \delta\left(x_{i}-c_{i}\right) d x_{i}$ is chosen as the generic measure in (2.2), leading to the recursive form

$$
\begin{aligned}
y(\mathbf{X}) & =\sum_{u \subseteq\{1, \cdots, N\}} y_{u, R}\left(\mathbf{X}_{u} ; \mathbf{c}\right), \\
y_{\emptyset, R} & =y(\mathbf{c}), \\
y_{u, R}\left(\mathbf{X}_{u} ; \mathbf{c}\right) & =y\left(\mathbf{X}_{u}, \mathbf{c}_{-u}\right)-\sum_{v \subset u} y_{v, R}\left(\mathbf{X}_{v} ; \mathbf{c}\right),
\end{aligned}
$$

presented as cut-HDMR [8, anchored decomposition [13, 14, 17, and anchoredANOVA decomposition [18, with the latter two referring to the reference point as the anchor. $\mathrm{Xu}$ and Rahman introduced (2.6) with the aid of Taylor series expansion, calling it dimension-reduction [10] and decomposition [11] methods for calculating statistical moments and reliability of mechanical system responses, respectively. Again, these various synonyms of the same decomposition exist due to diverse perspectives employed by researchers in disparate fields. Analogous to ADD, the RDD can also be described explicitly, for instance [13, 14, 17],

$$
y(\mathbf{X})=\sum_{u \subseteq\{1, \cdots, N\}} \sum_{v \subseteq u}(-1)^{|u|-|v|} y\left(\mathbf{X}_{v}, \mathbf{c}_{-v}\right),
$$

where $\left(\mathbf{X}_{v}, \mathbf{c}_{-v}\right)$ denotes an $N$-dimensional vector whose $i$ th component is $X_{i}$ if $i \in v$ and $c_{i}$ if $i \notin v$. The second argument "c " introduced in $y_{u, R}$ and $y_{v, R}$ is a reminder that the RDD component functions depend on the reference point, although $y$ does not, as (2.7) is exact.

An important property of the RDD component functions, also inherited from (2.1), is as follows [14.

Proposition 2.3. The $R D D$ component functions $y_{u, R}, \emptyset \neq u \subseteq\{1, \cdots, N\}$, vanish when any of its own variables $X_{i}$ with $i \in u$ takes on the value of $c_{i}$, i.e.,

$$
y_{u, R}\left(\mathbf{X}_{u} ; \mathbf{c}\right)=0 \text { whenever } X_{i}=c_{i} \text { for all } i \in u \text {. }
$$

Clearly, the RDD component functions lack orthogonal features with respect to the probability measure of $\mathbf{X}$, but are relatively easy to obtain as they only involve function evaluations at a chosen reference point. However, the RDD component functions can be orthogonal with respect to other inner products [14]. 
2.3. Truncation. When a dimensional decomposition, whether ADD or RDD or others, of a multivariate function is truncated by retaining only lower-dimensional terms, the result is an approximation. However, due to the special structure of the decomposition, the approximation is endowed with an error-minimizing property, described in Theorem 2.4

Theorem 2.4 ([8]). For a multivariate function $y: \mathbb{R}^{N} \rightarrow \mathbb{R}$ and $0 \leq S<N$, if

$$
\begin{aligned}
\hat{y}_{S}(\mathbf{X}) & =\sum_{\substack{u \subseteq\{1, \cdots, N\} \\
0 \leq|u| \leq S}} y_{u}\left(\mathbf{X}_{u}\right) \\
y_{\emptyset} & =\int_{\mathbb{R}^{N}} y(\mathbf{x}) w(\mathbf{x}) d \mathbf{x} \\
y_{u}\left(\mathbf{X}_{u}\right) & =\int_{\mathbb{R}^{N-|u|}} y\left(\mathbf{X}_{u}, \mathbf{x}_{-u}\right) w_{-u}\left(\mathbf{x}_{-u}\right) d \mathbf{x}_{-u}-\sum_{v \subset u} y_{v}\left(\mathbf{X}_{v}\right),
\end{aligned}
$$

represent an $S$-variate approximation, obtained by truncating at $0 \leq|u| \leq S$ the dimensional decomposition of $y(\mathbf{X})$ with respect to the generic measure $w(\mathbf{x}) d \mathbf{x}$, then the component functions $y_{u}, 0 \leq|u| \leq S$, are uniquely determined from

$$
\begin{aligned}
& \min _{y_{u}, 0 \leq|u| \leq S} \int_{\mathbb{R}^{N}}\left[y(\mathbf{x})-\sum_{\substack{u \subseteq\{1, \cdots, N\} \\
0 \leq|u| \leq S}} y_{u}\left(\mathbf{x}_{u}\right)\right]^{2} w(\mathbf{x}) d \mathbf{x}, \\
& \text { subject to } \int_{\mathbb{R}^{\prime}} y_{u}\left(\mathbf{x}_{u}\right) w_{i}\left(x_{i}\right) d x_{i}=0 \text { for } i \in u .
\end{aligned}
$$

Rabitz and Alis [8] proved this theorem on pages 202-207 of their paper. The $\mathcal{L}_{2}$ error in (2.9) committed by a truncated dimensional decomposition is minimized, but only for a specific measure. Given a measure, no other choices of the component functions of $\hat{y}_{S}$ will produce approximations that are better than the one derived from (2.8). However, different measures will create different truncated decompositions, resulting in distinct approximation errors. Therefore, selecting a measure is vitally important for determining the approximation quality of a dimensional decomposition.

\section{ADD APPROXIMATION}

3.1. Second-moment properties. The $S$-variate ADD approximation $\hat{y}_{S, A}(\mathbf{X})$, say, of $y(\mathbf{X})$, where $0 \leq S<N$, is obtained by truncating the right side of (2.4a) at $0 \leq|u| \leq S$, yielding

$$
\hat{y}_{S, A}(\mathbf{X})=\sum_{\substack{u \subseteq\{1, \cdots, N\} \\ 0 \leq|u| \leq S}} y_{u, A}\left(\mathbf{X}_{u}\right) .
$$

By applying the expectation operator on $y(\mathbf{X})$ and $\hat{y}_{S, A}(\mathbf{X})$ from (2.4a) and (3.1), respectively, and noting Proposition 2.1 the mean $\mathbb{E}\left[\hat{y}_{S, A}(\mathbf{X})\right]=y_{\emptyset, A}$ of the $S$ variate $\mathrm{ADD}$ approximation matches the exact mean $\mathbb{E}[y(\mathbf{X})]:=\int_{\mathbb{R}^{N}} y(\mathbf{x}) f_{\mathbf{X}}(\mathbf{x}) d \mathbf{x}$ $=y_{\emptyset, A}$, regardless of $S$. Applying the expectation operator again, this time on 
$\left(\hat{y}_{S, A}(\mathbf{X})-y_{\emptyset, A}\right)^{2}$, and recognizing Proposition 2.2 results in splitting the variance

$$
\hat{\sigma}_{S, A}^{2}:=\mathbb{E}\left[\left(\hat{y}_{S, A}(\mathbf{X})-y_{\emptyset, A}\right)^{2}\right]=\sum_{\substack{\emptyset \neq u \subseteq\{1, \cdots, N\} \\ 1 \leq|u| \leq S}} \sigma_{u}^{2}=\sum_{s=1}^{S} \sum_{\substack{\emptyset \neq u \subseteq\{1, \cdots, N\} \\|u|=s}} \sigma_{u}^{2}
$$

of the $S$-variate ADD approximation, where $\sigma_{u}^{2}:=\mathbb{E}\left[y_{u, A}^{2}\left(\mathbf{X}_{u}\right)\right]$ represents the variance of the zero-mean ADD component function $y_{u, A}, \emptyset \neq u \subseteq\{1, \cdots, N\}$. Clearly, the approximate variance in (3.2) approaches the exact variance

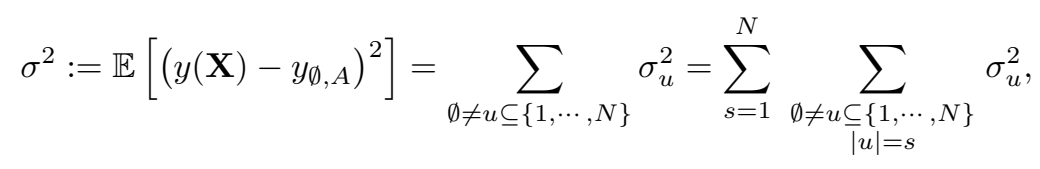

the sum of all variance terms, when $S \rightarrow N$. A normalized version $\sigma_{u}^{2} / \sigma^{2}$ is often called the global sensitivity index of $y$ for $\mathbf{X}_{u}$ [19.

3.2. Error from general approximation. Define a mean-squared error

$$
e_{S, A}:=\mathbb{E}\left[\left(y(\mathbf{X})-\hat{y}_{S, A}(\mathbf{X})\right)^{2}\right]:=\int_{\mathbb{R}^{N}}\left[y(\mathbf{x})-\hat{y}_{S, A}(\mathbf{x})\right]^{2} f_{\mathbf{X}}(\mathbf{x}) d \mathbf{x}
$$

committed by the $S$-variate ADD approximation $\hat{y}_{S, A}(\mathbf{X})$ of $y(\mathbf{X})$. Replacing $y$ and $\hat{y}_{S, A}$ in (3.3) with the right sides of (2.4a) and (3.1), respectively, and then recognizing Propositions 2.1 and 2.2 yields

$$
e_{S, A}=\sum_{\substack{\emptyset \neq u \subseteq\{1, \cdots, N\} \\ S+1 \leq|u| \leq N}} \sigma_{u}^{2}=\sum_{s=S+1}^{N} \sum_{\substack{\emptyset \neq u \subseteq\{1, \cdots, N\} \\|u|=s}} \sigma_{u}^{2},
$$

which completely eliminates the variance terms of $\sigma^{2}$ that are associated with $S$ and all lower-variate contributions, an attractive property of ADD. By setting $S=$ $0,1,2, \cdots$, the error can be expressed for any truncation of ADD.

3.3. Optimality. Among all possible measures, the probability measure endows the ADD approximation with an error-minimizing property, explained as follows.

Proposition 3.1. For a given $S$, the $S$-variate $A D D$ approximation is optimal in the mean-square sense.

Proof. Consider a generic $S$-variate approximation $\hat{y}_{S}(\mathbf{X})$ of $y(\mathbf{X})$ other than the ADD approximation $\hat{y}_{S, A}(\mathbf{X})$. Since $y(\mathbf{X})-\hat{y}_{S, A}(\mathbf{X})$ contains only higher than $S$ variate terms and $\hat{y}_{S, A}(\mathbf{X})-\hat{y}_{S}(\mathbf{X})$ contains at most $S$-variate terms, $y-\hat{y}_{S, A}$ and $\hat{y}_{S, A}-\hat{y}_{S}$ are orthogonal, satisfying $\mathbb{E}\left[\left(y(\mathbf{X})-\hat{y}_{S, A}(\mathbf{X})\right)\left(\hat{y}_{S, A}(\mathbf{X})-\hat{y}_{S}(\mathbf{X})\right)\right]=0$. Consequently, the second-moment error from an $S$-variate approximation

$$
\begin{aligned}
e_{S} & :=\mathbb{E}\left[\left(y(\mathbf{X})-\hat{y}_{S}(\mathbf{X})\right)^{2}\right] \\
& =\mathbb{E}\left[\left(y(\mathbf{X})-\hat{y}_{S, A}(\mathbf{X})\right)^{2}\right]+\mathbb{E}\left[\left(\hat{y}_{S, A}(\mathbf{X})-\hat{y}_{S}(\mathbf{X})\right)^{2}\right] \\
& =e_{S, A}+\mathbb{E}\left[\left(\hat{y}_{S, A}(\mathbf{X})-\hat{y}_{S}(\mathbf{X})\right)^{2}\right] \geq e_{S, A},
\end{aligned}
$$

proving the mean-square optimality of the $S$-variate ADD approximation. 
Therefore, given a truncation, an RDD approximation, regardless of how the reference point is selected, cannot be better than an ADD approximation for calculating variance. Further details of RDD approximation errors are described in the next section.

\section{RDD APPROXIMATION}

4.1. Direct form. The $S$-variate RDD approximation $\hat{y}_{S, R}(\mathbf{X} ; \mathbf{c})$, say, of $y(\mathbf{X})$, where $0 \leq S<N$, is obtained by truncating the right side of (2.6a) at $0 \leq|u| \leq S$, yielding

$$
\hat{y}_{S, R}(\mathbf{X} ; \mathbf{c})=\sum_{\substack{u \subseteq\{1, \cdots, N\} \\ 0 \leq|u| \leq S}} y_{u, R}\left(\mathbf{X}_{u} ; \mathbf{c}\right),
$$

which depends on the reference point, needing the second argument "c" in $\hat{y}_{S, R}$. For error analysis, however, a suitable direct form of (4.1) is desirable. Theorem 4.1 supplies such a form, which was originally obtained by $\mathrm{Xu}$ and Rahman 10 . using the Taylor series expansion. The same form was reported later by Kuo et al. 14.

Let $\mathbf{j}_{k}=\left(j_{1}, \cdots, j_{k}\right) \in \mathbb{N}_{0}^{k}, 1 \leq k \leq S$, be a $k$-dimensional multi-index with each component representing a non-negative integer. The multi-index, used in Theorem 4.1, obeys the following standard notations: (1) $\left|\mathbf{j}_{k}\right|=\sum_{p=1}^{p=k} j_{p} ;(2) \mathbf{j}_{k} !=\prod_{p=1}^{p=k} j_{p}$ !; (3) $\partial^{\mathbf{j}_{k}} y(\mathbf{c})=\partial^{j_{1}+\cdots+j_{k}} y(\mathbf{c}) / \partial X_{i_{1}}^{j_{1}} \cdots \partial X_{i_{k}}^{j_{k}} ;$ (4) $\left(\mathbf{X}_{u}-\mathbf{c}_{u}\right)^{\mathbf{j}_{k}}=\prod_{p=1}^{p=k}\left(X_{i_{p}}-c_{i_{p}}\right)^{j_{p}}$, $1 \leq i_{1}<\cdots<i_{k} \leq N$.

Theorem 4.1 (Multivariate Function Theorem [10]). For a differentiable multivariate function $y: \mathbb{R}^{N} \rightarrow \mathbb{R}$ and $0 \leq S<N$, if

$$
\hat{y}_{S, R}(\mathbf{X} ; \mathbf{c})=\sum_{k=0}^{S}(-1)^{k}\left(\begin{array}{c}
N-S+k-1 \\
k
\end{array}\right) \sum_{\substack{u \subseteq\{1, \cdots, N\} \\
|u|=S-k}} y\left(\mathbf{X}_{u}, \mathbf{c}_{-u}\right)
$$

represents an $S$-variate $R D D$ approximation of $y(\mathbf{X})$, then $\hat{y}_{S, R}(\mathbf{X} ; \mathbf{c})$ consists of all terms of the Taylor series expansion of $y(\mathbf{X})$ at $\mathbf{c}$ that have less than or equal to $S$ variables, i.e.,

where

$$
\hat{y}_{S, R}(\mathbf{X} ; \mathbf{c})=\sum_{k=0}^{S} t_{k}
$$

$$
\begin{aligned}
t_{0} & =y(\mathbf{c}), \\
t_{k} & =\sum_{\substack{\mathbf{j}_{k} \in \mathbb{N}_{0}^{k} \\
j_{1}, \cdots, j_{k} \neq 0}} \frac{1}{\mathbf{j}_{k} !} \sum_{\substack{\emptyset \neq u \subseteq\{1, \cdots, N\} \\
|u|=k}} \partial^{\mathbf{j}_{k}} y(\mathbf{c})\left(\mathbf{X}_{u}-\mathbf{c}_{u}\right)^{\mathbf{j}_{k}} ; 1 \leq k \leq S .
\end{aligned}
$$

$\mathrm{Xu}$ and Rahman [10] proved this theorem on pages 1996-2000 of their paper when $\mathbf{c}=\mathbf{0}$ without loss of generality. The stochastic method associated with the RDD approximation was simply called the "decomposition method" [11]. Theorem 4.1 implies that the RDD approximation $\hat{y}_{S, R}(\mathbf{X} ; \mathbf{c})$ in (4.2), when compared with the Taylor series expansion of $y(\mathbf{X})$, yields a residual error that includes only terms of dimensions $S+1$ and higher. All higher-order $S$ - and lower-variate terms of 
$y(\mathbf{X})$ are included in (4.2), which should therefore generally provide a higher-order approximation of a multivariate function than the equation derived from an $S$-order Taylor expansion.

Equations (4.1) and (4.2) both follow the same structure as (1.2). However, due to the distinct perspectives involved, it is not obvious if these equations represent the same function $\hat{y}_{S, R}$. A lemma and a theorem recently proved by the author demonstrate that, indeed, they do [20].

Corollary 4.2. When $S=0,1$, and 2 , (4.2) degenerates to the zero-variate $R D D$ approximation

$$
\hat{y}_{0, R}(\mathbf{X} ; \mathbf{c})=y(\mathbf{c})
$$

the univariate $R D D$ approximation

$$
\hat{y}_{1, R}(\mathbf{X} ; \mathbf{c})=\sum_{i=1}^{N} y\left(X_{i}, \mathbf{c}_{-\{i\}}\right)-(N-1) y(\mathbf{c}),
$$

and the bivariate $R D D$ approximation

$$
\begin{aligned}
\hat{y}_{2, R}(\mathbf{X} ; \mathbf{c})= & \sum_{i=1}^{N-1} \sum_{j=i+1}^{N} y\left(X_{i}, X_{j}, \mathbf{c}_{-\{i, j\}}\right)-(N-2) \sum_{i=1}^{N} y\left(X_{i}, \mathbf{c}_{-\{i\}}\right) \\
& +\frac{1}{2}(N-1)(N-2) y(\mathbf{c}),
\end{aligned}
$$

respectively.

Remark 4.3. Since the right side of (4.4) comprises only univariate functions, the interpolation or integration of $\hat{y}_{1, R}(\mathbf{X} ; \mathbf{c})$ is essentially univariate. Similarly, the right side of (4.5), which contains at most bivariate functions, requires at most bivariate interpolation or integration of $\hat{y}_{2, R}(\mathbf{X} ; \mathbf{c})$. Therefore, appellation of the terms "univariate approximation" and "bivariate approximation" for $\hat{y}_{1, R}(\mathbf{X} ; \mathbf{c})$ in (4.4) and $\hat{y}_{2, R}(\mathbf{X} ; \mathbf{c})$ in (4.5), respectively, is more appropriate than referring to them as first-order and second-order approximations.

4.2. Expected error. Following similar consideration, define another meansquared error

$$
e_{S, R}(\mathbf{c}):=\mathbb{E}\left[\left(y(\mathbf{X})-\hat{y}_{S, R}(\mathbf{X} ; \mathbf{c})\right)^{2}\right]:=\int_{\mathbb{R}^{N}}\left[y(\mathbf{x})-\hat{y}_{S, R}(\mathbf{x} ; \mathbf{c})\right]^{2} f_{\mathbf{X}}(\mathbf{x}) d \mathbf{x}
$$

associated with the $S$-variate RDD approximation $\hat{y}_{S, R}(\mathbf{X} ; \mathbf{c})$ of $y(\mathbf{X})$, which depends on the reference point $\mathbf{c}$. Wang [13] suggested choosing a random reference point uniformly distributed over $[0,1]^{N}$ and then calculating the error on average. But, $\mathbf{X}$ defined here may follow an arbitrary probability law with density $f_{\mathbf{X}}(\mathbf{x})$. Therefore, selecting the reference point characterized by the probability density $f_{\mathbf{X}}(\mathbf{c})$ is more appropriate, which leads to

$$
\begin{aligned}
\mathbb{E}\left[e_{S, R}(\mathbf{c})\right] & :=\int_{\mathbb{R}^{N}} e_{S, R}(\mathbf{c}) f_{\mathbf{X}}(\mathbf{c}) d \mathbf{c} \\
& =\int_{\mathbb{R}^{2 N}}\left[y(\mathbf{x})-\hat{y}_{S, R}(\mathbf{x} ; \mathbf{c})\right]^{2} f_{\mathbf{X}}(\mathbf{x}) f_{\mathbf{X}}(\mathbf{c}) d \mathbf{x} d \mathbf{c}
\end{aligned}
$$

as the expected value of the RDD error. Simplifying (4.7) in terms of the variance components of $y$, as done for the ADD error in (3.4), for arbitrary $S$ and $N$ may 
appear formidable. Here, the zero-variate $(S=0)$, univariate $(S=1)$, and bivariate $(S=2)$ approximation errors for arbitrary $N$ will be derived first, followed by error analysis for a general $S$-variate approximation. In all cases, the derivations require using (1) the relationships,

$$
\begin{aligned}
y_{\emptyset, A} & =\int_{\mathbb{R}^{N}} y(\mathbf{c}) f_{\mathbf{X}}(\mathbf{c}) d \mathbf{c} \\
y_{u, A}\left(\mathbf{x}_{u}\right) & =\int_{\mathbb{R}^{N}} y_{u, R}\left(\mathbf{x}_{u} ; \mathbf{c}\right) f_{\mathbf{X}}(\mathbf{c}) d \mathbf{c} \\
\hat{y}_{S, A}(\mathbf{x}) & =\int_{\mathbb{R}^{N}} \hat{y}_{S, R}(\mathbf{x} ; \mathbf{c}) f_{\mathbf{X}}(\mathbf{c}) d \mathbf{c}
\end{aligned}
$$

that exist between ADD and RDD component functions and approximations and

(2) Sobol's formula [19]

$$
D_{u}:=\sum_{v \subseteq u} \sigma_{v}^{2}=\int_{\mathbb{R}^{2 N-|u|}} y(\mathbf{x}) y\left(\mathbf{x}_{u}, \mathbf{c}_{-u}\right) f_{\mathbf{X}}(\mathbf{x}) f_{\mathbf{X}_{-u}}\left(\mathbf{c}_{-u}\right) d \mathbf{x} d \mathbf{c}_{-u}-y_{\emptyset, A}^{2}
$$

for select choices of $u$ described in the following subsection. Equations 4.8a and (4.8b) follow from Propositions 2.1, 2.2, and 2.3 and definitions of respective component functions in (2.4) and (2.6), eventually leading to (4.8c). The term $D_{u}$ in Sobol's formula represents a sum of variance terms contributed by the ADD component functions that belong to $\emptyset \neq u \subseteq\{1, \cdots, N\}$.

4.3. Expected errors from zero-variate, univariate, and bivariate approximations. Theorems 4.4, 4.5, and 4.6 show how the expected errors from the zero-variate, univariate, and bivariate RDD approximations, respectively, depend on the variance components of $y$.

Theorem 4.4. Let $\mathbf{c}=\left(c_{1}, \cdots, c_{N}\right) \in \mathbb{R}^{N}$ be a random vector with the joint probability density function of the form $f_{\mathbf{X}}(\mathbf{c})=\prod_{j=1}^{j=N} f_{j}\left(c_{j}\right)$, where $f_{j}$ is the marginal probability density function of its $j$ th coordinate. Then the expected error committed by the zero-variate $R D D$ approximation for $1 \leq N<\infty$ is

$$
\mathbb{E}\left[e_{0, R}(\mathbf{c})\right]=2 \sigma^{2},
$$

where $\sigma^{2}:=\mathbb{E}\left[\left(y(\mathbf{X})-y_{\emptyset, A}\right)^{2}\right]=\mathbb{E}\left[y^{2}(\mathbf{X})\right]-y_{\emptyset, A}^{2}$ is the variance of $y$.

Proof. Setting $S=0$ in (4.7) and using the expression of $\hat{y}_{0, R}(\mathbf{x} ; \mathbf{c})$ from (4.3), the expected error from the zero-variate RDD approximation becomes

$$
\begin{aligned}
\mathbb{E}\left[e_{0, R}(\mathbf{c})\right]= & \int_{\mathbb{R}^{2 N}}[y(\mathbf{x})-y(\mathbf{c})]^{2} f_{\mathbf{X}}(\mathbf{x}) f_{\mathbf{X}}(\mathbf{c}) d \mathbf{x} d \mathbf{c} \\
= & \int_{\mathbb{R}^{N}} y^{2}(\mathbf{x}) f_{\mathbf{X}}(\mathbf{x}) d \mathbf{x}+\int_{\mathbb{R}^{N}} y^{2}(\mathbf{c}) f_{\mathbf{X}}(\mathbf{c}) d \mathbf{c} \\
& -2 \int_{\mathbb{R}^{N}} y(\mathbf{x}) f_{\mathbf{X}}(\mathbf{x}) d \mathbf{x} \int_{\mathbb{R}^{N}} y(\mathbf{c}) f_{\mathbf{X}}(\mathbf{c}) d \mathbf{c} \\
= & \sigma^{2}+y_{\emptyset, A}^{2}+\sigma^{2}+y_{\emptyset, A}^{2}-2 y_{\emptyset, A}^{2} \\
= & 2 \sigma^{2}
\end{aligned}
$$

where the third equality exploits the ADD-RDD relationship in (4.8a). Hence, the theorem is proven. 
Theorem 4.5. Let $\mathbf{c}=\left(c_{1}, \cdots, c_{N}\right) \in \mathbb{R}^{N}$ be a random vector with the joint probability density function of the form $f_{\mathbf{X}}(\mathbf{c})=\prod_{j=1}^{j=N} f_{j}\left(c_{j}\right)$, where $f_{j}$ is the marginal probability density function of its $j$ th coordinate. Then the expected error committed by the univariate $R D D$ approximation for $2 \leq N<\infty$ is

$$
\mathbb{E}\left[e_{1, R}(\mathbf{c})\right]=\sum_{s=2}^{N}\left(s^{2}-s+2\right) \sum_{\substack{\emptyset \neq u \subseteq\{1, \cdots, N\} \\|u|=s}} \sigma_{u}^{2},
$$

where $\sigma_{u}^{2}=\mathbb{E}\left[y_{u, A}^{2}\left(\mathbf{X}_{u}\right)\right]$ is the variance of the zero-mean ADD component function $y_{u, A}, \emptyset \neq u \subseteq\{1, \cdots, N\}$.

Proof. Setting $S=1$ in (4.7), the expected error from the univariate RDD approximation on expansion is a sum

$$
\mathbb{E}\left[e_{1, R}(\mathbf{c})\right]=I_{1,1}+I_{1,2}+I_{1,3}
$$

of three integrals

$$
\begin{aligned}
I_{1,1} & :=\int_{\mathbb{R}^{2 N}} y^{2}(\mathbf{x}) f_{\mathbf{X}}(\mathbf{x}) f_{\mathbf{X}}(\mathbf{c}) d \mathbf{x} d \mathbf{c} \\
I_{1,2} & :=-2 \int_{\mathbb{R}^{2 N}} y(\mathbf{x}) \hat{y}_{1, R}(\mathbf{x} ; \mathbf{c}) f_{\mathbf{X}}(\mathbf{x}) f_{\mathbf{X}}(\mathbf{c}) d \mathbf{x} d \mathbf{c}, \\
I_{1,3} & :=\int_{\mathbb{R}^{2 N}} \hat{y}_{1, R}^{2}(\mathbf{x} ; \mathbf{c}) f_{\mathbf{X}}(\mathbf{x}) f_{\mathbf{X}}(\mathbf{c}) d \mathbf{x} d \mathbf{c}
\end{aligned}
$$

on $\mathbb{R}^{2 N}$, where their first indices represent the univariate approximation. The first integral

$$
I_{1,1}=\mathbb{E}\left[y^{2}(\mathbf{X})\right]=y_{\emptyset, A}^{2}+\sigma^{2}=y_{\emptyset, A}^{2}+\sum_{s=1}^{N} \sum_{\substack{\emptyset \neq u \subseteq\{1, \cdots, N\} \\|u|=s}} \sigma_{u}^{2},
$$

expressed in terms of the variance components, is independent of $S$. However, the second integral depends on $S$, yielding

$$
\begin{aligned}
I_{1,2} & =-2 \int_{\mathbb{R}^{N}} y(\mathbf{x}) \hat{y}_{1, A}(\mathbf{x}) f_{\mathbf{X}}(\mathbf{x}) d \mathbf{x} \\
& =-2 \int_{\mathbb{R}^{N}} \hat{y}_{1, A}^{2}(\mathbf{x}) f_{\mathbf{X}}(\mathbf{x}) d \mathbf{x} \\
& =-2 \mathbb{E}\left[\hat{y}_{1, A}^{2}(\mathbf{X})\right] \\
& =-2\left(y_{\emptyset, A}^{2}+\hat{\sigma}_{1, A}^{2}\right) \\
& =-2 y_{\emptyset, A}^{2}-2 \sum_{\emptyset \neq u \subseteq\{1, \cdots, N\}} \sigma_{u}^{2},
\end{aligned}
$$

where the first, second, and fifth lines are obtained by (1) employing the ADD-RDD relationships in (4.8c) for $S=1,(2)$ recognizing $y-\hat{y}_{1, A}$ and $\hat{y}_{1, A}$ to be orthogonal, satisfying $\mathbb{E}\left[\left(y(\mathbf{X})-\hat{y}_{1, A}(\mathbf{X})\right) \hat{y}_{1, A}(\mathbf{X})\right]=0$, and (3) applying (3.2) for $S=1$, respectively. Using the expression of $\hat{y}_{1, R}(\mathbf{x} ; \mathbf{c})$ from (4.4) and noting independent 
coordinates of $\mathbf{X}$ and $\mathbf{c}$, the expanded third integral becomes

$$
\begin{aligned}
I_{1,3}= & \int_{\mathbb{R}^{2 N}}\left[\sum_{i=1}^{N} y\left(x_{i}, \mathbf{c}_{-\{i\}}\right)-(N-1) y(\mathbf{c})\right]^{2} f_{\mathbf{X}}(\mathbf{x}) f_{\mathbf{X}}(\mathbf{c}) d \mathbf{x} d \mathbf{c} \\
= & \int_{\mathbb{R}^{2 N}}\left[\sum_{i=1}^{N} y^{2}\left(x_{i}, \mathbf{c}_{-\{i\}}\right)+2 \sum_{i=1}^{N-1} \sum_{j=i+1}^{N} y\left(x_{i}, \mathbf{c}_{-\{i\}}\right) y\left(x_{j}, \mathbf{c}_{-\{j\}}\right)\right. \\
& \left.+(N-1)^{2} y^{2}(\mathbf{c})-2(N-1) \sum_{i=1}^{N} y\left(X_{i}, \mathbf{c}_{-\{i\}}\right) y(\mathbf{c})\right] f_{\mathbf{X}}(\mathbf{x}) f_{\mathbf{X}}(\mathbf{c}) d \mathbf{x} d \mathbf{c} .
\end{aligned}
$$

Further evaluation of this integral requires exploiting Sobol's formula in (4.9) for $u=-\{i\}$ and $u=-\{i, j\}$, where $i, j=1, \cdots, N, i<j$, yielding

$$
\begin{aligned}
& I_{1,3}=\left(N^{2}-N+1\right)\left(\sigma^{2}+y_{\emptyset, A}^{2}\right)-2(N-1) \sum_{i=1}^{N}\left(D_{-\{i\}}+y_{\emptyset, A}^{2}\right) \\
& +2 \sum_{i=1}^{N-1} \sum_{j=i+1}^{N}\left(D_{-\{i, j\}}+y_{\emptyset, A}^{2}\right) \\
& =y_{\emptyset, A}^{2}+\sum_{\substack{\emptyset \neq u \subseteq\{1, \cdots, N\} \\
|u|=1}} \sigma_{u}^{2}+3 \sum_{\substack{\emptyset \neq u \subseteq\{1, \cdots, N\} \\
|u|=2}} \sigma_{u}^{2} \\
& +\cdots+\left(s^{2}-s+1\right) \sum_{\substack{\emptyset \neq u \subseteq\{1, \cdots, N\} \\
|u|=s}} \sigma_{u}^{2}+\cdots+\left(N^{2}-N+1\right) \sum_{\substack{\emptyset \neq u \subseteq\{1, \cdots, N\} \\
|u|=N}} \sigma_{u}^{2} \\
& =b_{1}(0) y_{\emptyset, A}^{2}+\sum_{s=1}^{N} b_{1}(s) \sum_{\substack{\emptyset \neq u \subseteq\{1, \cdots, N\} \\
|u|=s}} \sigma_{u}^{2},
\end{aligned}
$$

where

$$
b_{1}(s)=s^{2}-s+1,0 \leq s \leq N,
$$

is the generic $s$-variate coefficient for the univariate approximation, obtained by counting the number of $y_{\emptyset, A}^{2}$ or $\sigma_{u}^{2}$ for $|u|=s$ (e.g., $\sigma_{1}^{2}$ for $s=1, \sigma_{12}^{2}$ for $s=2$, and so on) due to symmetry. Adding all terms in (4.11), (4.12), and (4.14), with the recognition that $b_{1}(0)=b_{1}(1)=1$, yields (4.10), completing the proof.

Theorem 4.6. Let $\mathbf{c}=\left(c_{1}, \cdots, c_{N}\right) \in \mathbb{R}^{N}$ be a random vector with the joint probability density function of the form $f_{\mathbf{X}}(\mathbf{c})=\prod_{j=1}^{j=N} f_{j}\left(c_{j}\right)$, where $f_{j}$ is the marginal probability density function of its $j$ th coordinate. Then the expected error committed by the bivariate $R D D$ approximation for $3 \leq N<\infty$ is

$$
\mathbb{E}\left[e_{2, R}(\mathbf{c})\right]=\sum_{s=3}^{N} \frac{1}{4}\left(s^{4}-2 s^{3}-s^{2}+2 s+8\right) \sum_{\substack{\emptyset \neq u \subseteq\{1, \cdots, N\} \\|u|=s}} \sigma_{u}^{2},
$$

where $\sigma_{u}^{2}=\mathbb{E}\left[y_{u, A}^{2}\left(\mathbf{X}_{u}\right)\right]$ is the variance of the zero-mean ADD component function $y_{u, A}, \emptyset \neq u \subseteq\{1, \cdots, N\}$.

Proof. Setting $S=2$ in (4.7), the expected error from the bivariate RDD approximation on expansion is another sum

$$
\mathbb{E}\left[e_{2, R}(\mathbf{c})\right]=I_{2,1}+I_{2,2}+I_{2,3}
$$


of three $2 N$-dimensional integrals

$$
\begin{aligned}
I_{2,1} & :=\int_{\mathbb{R}^{2 N}} y^{2}(\mathbf{x}) f_{\mathbf{X}}(\mathbf{x}) f_{\mathbf{X}}(\mathbf{c}) d \mathbf{x} d \mathbf{c} \\
I_{2,2} & :=-2 \int_{\mathbb{R}^{2 N}} y(\mathbf{x}) \hat{y}_{2, R}(\mathbf{x} ; \mathbf{c}) f_{\mathbf{X}}(\mathbf{x}) f_{\mathbf{X}}(\mathbf{c}) d \mathbf{x} d \mathbf{c} \\
I_{2,3} & :=\int_{\mathbb{R}^{2 N}} \hat{y}_{2, R}^{2}(\mathbf{x} ; \mathbf{c}) f_{\mathbf{X}}(\mathbf{x}) f_{\mathbf{X}}(\mathbf{c}) d \mathbf{x} d \mathbf{c}
\end{aligned}
$$

where their first indices represent the bivariate approximation. Since the first integral does not depend on $S$,

$$
I_{2,1}=y_{\emptyset, A}^{2}+\sigma^{2}=y_{\emptyset, A}^{2}+\sum_{s=1}^{N} \sum_{\substack{\emptyset \neq u \subseteq\{1, \cdots, N\} \\|u|=s}} \sigma_{u}^{2}
$$

is the same as $I_{1,1}$. Following a similar reasoning employed for the univariate approximation, the second integral

$$
\begin{aligned}
I_{2,2} & =-2 \int_{\mathbb{R}^{N}} y(\mathbf{x}) \hat{y}_{2, A}(\mathbf{x}) f_{\mathbf{X}}(\mathbf{x}) d \mathbf{x} \\
& =-2 \int_{\mathbb{R}^{N}} \hat{y}_{2, A}^{2}(\mathbf{x}) f_{\mathbf{X}}(\mathbf{x}) d \mathbf{x} \\
& =-2 \mathbb{E}\left[\hat{y}_{2, A}^{2}(\mathbf{X})\right]=-2\left(y_{\emptyset, A}^{2}+\hat{\sigma}_{2, A}^{2}\right) \\
& =-2 y_{\emptyset, A}^{2}-2 \sum_{\emptyset \neq u \subseteq\{1, \cdots, N\}} \sigma_{u}^{2}-2 \sum_{\emptyset \neq u \subseteq\{1, \cdots, N\}} \sigma_{u}^{2}
\end{aligned}
$$

contains variance terms associated with at most two variables. Using the expression of $\hat{y}_{2, R}(\mathbf{x} ; \mathbf{c})$ from (4.5), the expanded third integral becomes

$$
\begin{aligned}
I_{2,3}= & \int_{\mathbb{R}^{2 N}}\left[\sum_{i=1}^{N-1} \sum_{j=i+1}^{N} y\left(x_{i}, x_{j}, \mathbf{c}_{-\{i, j\}}\right)-(N-2) \sum_{i=1}^{N} y\left(x_{i}, \mathbf{c}_{-\{i\}}\right)\right. \\
& \left.+\frac{1}{2}(N-1)(N-2) y(\mathbf{c})\right]^{2} f_{\mathbf{X}}(\mathbf{x}) f_{\mathbf{X}}(\mathbf{c}) d \mathbf{x} d \mathbf{c} \\
= & \int_{\mathbb{R}^{2 N}}\left\{\left[\sum_{i=1}^{N-1} \sum_{j=i+1}^{N} y\left(x_{i}, x_{j}, \mathbf{c}_{-\{i, j\}}\right)\right]^{2}+(N-2)^{2}\left[\sum_{i=1}^{N} y\left(x_{i}, \mathbf{c}_{-\{i\}}\right)\right]^{2}\right. \\
& +\frac{1}{4}(N-1)^{2}(N-2)^{2} y^{2}(\mathbf{c}) \\
& -2(N-2)\left[\sum_{i=1}^{N-1} \sum_{j=i+1}^{N} y\left(x_{i}, x_{j}, \mathbf{c}_{-\{i, j\}}\right)\right]\left[\sum_{i=1}^{N} y\left(x_{i}, \mathbf{c}_{-\{i\}}\right)\right] \\
& -(N-1)(N-2)^{2}\left[\sum_{i=1}^{N} y\left(x_{i}, \mathbf{c}_{-\{i\}}\right)\right] y(\mathbf{c}) \\
& \left.+(N-1)(N-2)\left[\sum_{i=1}^{N-1} \sum_{j=i+1}^{N} y\left(x_{i}, x_{j}, \mathbf{c}_{-\{i, j\}}\right)\right] y(\mathbf{c})\right\} f_{\mathbf{X}}(\mathbf{x}) f_{\mathbf{X}}(\mathbf{c}) d \mathbf{x} d \mathbf{c} .
\end{aligned}
$$


Employing Sobol's formula, this time for $u=-\{i\}, u=-\{j\}, u=-\{i, j\}, u=$ $-\{i, k\}, u=-\{j, k\}, u=-\{i, j, k\}$, and $u=-\{i, j, k, l\}$, where $i, j, k, l=1, \cdots, N$, $i<j<k<l$, results in

$$
\begin{aligned}
& I_{2,3}=\frac{N(N-1)}{2}\left(\sigma^{2}+y_{\emptyset, A}^{2}\right) \\
& +2 \sum_{i=1}^{N-2} \sum_{j=i+1}^{N-1} \sum_{k=j+1}^{N}\left(D_{-\{i, j\}}+D_{-\{i, k\}}+D_{-\{j, k\}}+3 y_{\emptyset, A}^{2}\right) \\
& +6 \sum_{i=1}^{N-3} \sum_{j=i+1}^{N-2} \sum_{k=j+1}^{N-1} \sum_{l=k+1}^{N}\left(D_{-\{i, j, k, l\}}+y_{\emptyset, A}^{2}\right)+N(N-2)^{2}\left(\sigma^{2}+y_{\emptyset, A}^{2}\right) \\
& +2(N-2)^{2} \sum_{i=1}^{N-1} \sum_{j=i+1}^{N}\left(D_{-\{i, j\}}+y_{\emptyset, A}^{2}\right)+\frac{1}{4}(N-1)^{2}(N-2)^{2}\left(\sigma^{2}+y_{\emptyset, A}^{2}\right) \\
& -2(N-2) \sum_{i=1}^{N-1} \sum_{j=i+1}^{N}\left(D_{-\{i\}}+D_{-\{j\}}+2 y_{\emptyset, A}^{2}\right) \\
& -6(N-2) \sum_{i=1}^{N-2} \sum_{j=i+1}^{N-1} \sum_{k=j+1}^{N}\left(D_{-\{i, j, k\}}+y_{\emptyset, A}^{2}\right) \\
& -(N-1)(N-2)^{2} \sum_{i=1}^{N}\left(D_{-\{i\}}+y_{\emptyset, A}^{2}\right) \\
& +(N-1)(N-2) \sum_{i=1}^{N-1} \sum_{j=i+1}^{N}\left(D_{-\{i, j\}}+y_{\emptyset, A}^{2}\right) \\
& =y_{\emptyset, A}^{2}+\sum_{\substack{\emptyset \neq u \subseteq\{1, \cdots, N\} \\
|u|=1}} \sigma_{u}^{2}+\sum_{\substack{\emptyset \neq u \subseteq\{1, \cdots, N\} \\
|u|=2}} \sigma_{u}^{2}+7 \sum_{\substack{\emptyset \neq u \subseteq\{1, \cdots, N\} \\
|u|=3}} \sigma_{u}^{2} \\
& +\cdots+\frac{1}{4}\left(s^{4}-2 s^{3}-s^{2}+2 s+4\right) \sum_{\substack{\emptyset \neq u \subseteq\{1, \cdots, N\} \\
|u|=s}} \sigma_{u}^{2} \\
& +\cdots+\frac{1}{4}\left(N^{4}-2 N^{3}-N^{2}+2 N+4\right) \sum_{\substack{\emptyset \neq u \subseteq\{1, \cdots, N\} \\
|u|=N}} \sigma_{u}^{2} \\
& =b_{2}(0) y_{\emptyset, A}^{2}+\sum_{s=1}^{N} b_{2}(s) \sum_{\substack{\emptyset \neq u \subseteq\{1, \cdots, N\} \\
|u|=s}} \sigma_{u}^{2},
\end{aligned}
$$

producing the generic $s$-variate coefficient

$$
b_{2}(s)=\frac{1}{4}\left(s^{4}-2 s^{3}-s^{2}+2 s+4\right), 0 \leq s \leq N,
$$

for the bivariate approximation. Adding all terms in (4.16), (4.17), and (4.18), with the understanding that $b_{2}(0)=b_{2}(1)=b_{2}(2)=1$, yields (4.15), proving the theorem. 
Corollary 4.7. The expected error $\mathbb{E}\left[e_{0, R}\right]$ from the zero-variate $R D D$ approximation, expressed in terms of the error $e_{0, A}$ from the zero-variate $A D D$ approximation, is

$$
\mathbb{E}\left[e_{0, R}\right]=2 e_{0, A}, 1 \leq N<\infty .
$$

Corollary 4.8. The lower and upper bounds of the expected errors $\mathbb{E}\left[e_{1, R}\right]$ and $\mathbb{E}\left[e_{2, R}\right]$ from the univariate and bivariate $R D D$ approximations, respectively, expressed in terms of the errors $e_{1, A}$ and $e_{2, A}$ from the univariate and bivariate $A D D$ approximations, are

$$
4 e_{1, A} \leq \mathbb{E}\left[e_{1, R}\right] \leq\left(N^{2}-N+2\right) e_{1, A}, 2 \leq N<\infty,
$$

and

$$
8 e_{2, A} \leq \mathbb{E}\left[e_{2, R}\right] \leq \frac{1}{4}\left(N^{4}-2 N^{3}-N^{2}+2 N+8\right) e_{2, A}, 3 \leq N<\infty,
$$

respectively.

Remark 4.9. When $\mathbf{X}$ comprises independent and identically distributed uniform random variables over $[0,1]$, the results of the zero-variate and univariate RDD approximations presented in Theorems 4.4 and 4.5 coincide with those derived by Wang 13. However, the results of the bivariate RDD approximation - that is, Theorem 4.6 - are new. Theorems 4.5 and 4.6 demonstrate that on average the error from the univariate RDD approximation eliminates the variance terms associated with the univariate contribution. For the bivariate RDD approximation, the variance portions resulting from the univariate and bivariate terms have been removed as well. The univariate and bivariate ADD approximations also satisfy this important property. However, the coefficients of higher-variate terms in the RDD errors are larger than unity, implying greater errors from RDD approximations than from ADD approximations.

Remark 4.10. From Corollary 4.7, the zero-variate RDD approximation on average commits twice as many errors as does the zero-variate ADD approximation. Since a zero-variate approximation, whether derived from ADD or RDD, does not capture the random fluctuations of a stochastic response, the error analysis associated with a zero-variate approximation is useless. Nonetheless, the zero-variate results are reported here for completeness.

Remark 4.11. Corollary 4.8 shows that the expected error from the univariate RDD approximation is at least four times larger than the error from the univariate ADD approximation. In contrast, the expected error from the bivariate RDD approximation can be eight times larger or more than the error from the bivariate ADD approximation. Given a truncation, an ADD approximation is superior to an RDD approximation. In addition, RDD approximations may perpetrate very large errors at upper bounds when there exist a large number of variables and appropriate conditions. For instance, consider a contrived example involving a function of 100 variables with a finite variance $\sigma^{2}>0$ and the following distribution of the variance terms: $\sum_{|u|=1} \sigma_{u}^{2}=0.999 \sigma^{2}, \sum_{2 \leq|u| \leq 99} \sigma_{u}^{2}=0$, and $\sum_{|u|=100} \sigma_{u}^{2}=0.001 \sigma^{2}$. Then, the errors from the univariate and bivariate ADD approximations are both equal to $0.001 \sigma^{2}$, which is negligibly small. In contrast, the error from the univariate RDD approximation reaches $\left(100^{2}-100+2\right) \times 0.001 \sigma^{2} \cong 9.9 \sigma^{2}$, an unacceptably 
large magnitude already. Furthermore, the error from the bivariate RDD approximation jumps to an enormously large value of $\frac{1}{4}\left(100^{4}-2 \times 100^{3}-100^{2}+2 \times\right.$ $100+8) \times 0.001 \sigma^{2} \cong 24,498 \sigma^{2}$. More importantly, the results reveal a theoretical possibility for a higher-variate RDD approximation to commit a larger error than a lower-variate RDD approximation - an impossible scenario for the ADD approximation. However, it is unlikely for this odd behavior to be exhibited for realistic functions, where the variances of higher-variate component functions attenuate rapidly or vanish altogether. Nonetheless, caution is warranted when employing RDD approximations for stochastic analysis of high-dimensional systems.

4.4. Expected error from general approximation. The error analysis presented so far is limited to at most the bivariate approximation. In this subsection, the approximation error from a general $S$-variate truncation is derived as follows.

Theorem 4.12. Let $\mathbf{c}=\left(c_{1}, \cdots, c_{N}\right) \in \mathbb{R}^{N}$ be a random vector with the joint probability density function of the form $f_{\mathbf{X}}(\mathbf{c})=\prod_{j=1}^{j=N} f_{j}\left(c_{j}\right)$, where $f_{j}$ is the marginal probability density function of its $j$ th coordinate. Then the expected error committed by the $S$-variate $R D D$ approximation for $0 \leq S<N, S+1 \leq N<\infty$ is

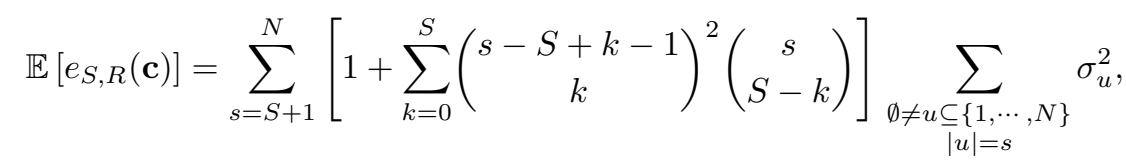

where $\sigma_{u}^{2}=\mathbb{E}\left[y_{u, A}^{2}\left(\mathbf{X}_{u}\right)\right]$ is the variance of the zero-mean ADD component function $y_{u, A}, \emptyset \neq u \subseteq\{1, \cdots, N\}$.

Proof. Expanding the square in (4.7), the expected error from the $S$-variate RDD approximation is

$$
\mathbb{E}\left[e_{S, R}(\mathbf{c})\right]=I_{S, 1}+I_{S, 2}+I_{S, 3},
$$

where

$$
\begin{aligned}
I_{S, 1} & :=\int_{\mathbb{R}^{2 N}} y^{2}(\mathbf{x}) f_{\mathbf{X}}(\mathbf{x}) f_{\mathbf{X}}(\mathbf{c}) d \mathbf{x} d \mathbf{c} \\
I_{S, 2} & :=-2 \int_{\mathbb{R}^{2 N}} y(\mathbf{x}) \hat{y}_{S, R}(\mathbf{x} ; \mathbf{c}) f_{\mathbf{X}}(\mathbf{x}) f_{\mathbf{X}}(\mathbf{c}) d \mathbf{x} d \mathbf{c}, \\
I_{S, 3} & :=\int_{\mathbb{R}^{2 N}} \hat{y}_{S, R}^{2}(\mathbf{x} ; \mathbf{c}) f_{\mathbf{X}}(\mathbf{x}) f_{\mathbf{X}}(\mathbf{c}) d \mathbf{x} d \mathbf{c}
\end{aligned}
$$

are three generic $2 N$-dimensional integrals. The first integral,

$$
I_{S, 1}=y_{\emptyset, A}^{2}+\sigma^{2}=y_{\emptyset, A}^{2}+\sum_{s=1}^{N} \sum_{\substack{\emptyset \neq u \subseteq\{1, \cdots, N\} \\|u|=s}} \sigma_{u}^{2},
$$


is the same as before. The second integral,

$$
\begin{aligned}
& I_{S, 2}=-2 \int_{\mathbb{R}^{N}} y(\mathbf{x}) \hat{y}_{S, A}(\mathbf{x}) f_{\mathbf{X}}(\mathbf{x}) d \mathbf{x} \\
& =-2 \int_{\mathbb{R}^{N}} \hat{y}_{S, A}^{2}(\mathbf{x}) f_{\mathbf{X}}(\mathbf{x}) d \mathbf{x} \\
& =-2 \mathbb{E}\left[\hat{y}_{S, A}^{2}(\mathbf{X})\right]=-2\left(y_{\emptyset, A}^{2}+\hat{\sigma}_{S, A}^{2}\right) \\
& =-2 y_{\emptyset, A}^{2}-2 \sum_{s=1}^{S} \sum_{\substack{\emptyset \neq u \subseteq\{1, \cdots, N\} \\
|u|=s}} \sigma_{u}^{2} \text {, }
\end{aligned}
$$

comprises variance terms associated with at most $S$ variables. The third integral, using $\hat{y}_{S, R}(\mathbf{x} ; \mathbf{c})$ from (4.2), takes the form

$$
\begin{aligned}
I_{S, 3} & =\int_{\mathbb{R}^{2 N}}\left[\sum_{k=0}^{S}(-1)^{k}\left(\begin{array}{c}
N-S+k-1 \\
k
\end{array}\right) \sum_{\substack{u \subseteq\{1, \cdots, N\} \\
|u|=S-k}} y\left(\mathbf{x}_{u}, \mathbf{c}_{-u}\right)\right]^{2} f_{\mathbf{X}}(\mathbf{x}) f_{\mathbf{X}}(\mathbf{c}) d \mathbf{x} d \mathbf{c} \\
& =b_{S}(0) y_{\emptyset, A}^{2}+\sum_{s=1}^{N} b_{S}(s) \sum_{\substack{\emptyset \neq u \subseteq\{1, \cdots, N\} \\
|u|=s}} \sigma_{u}^{2}
\end{aligned}
$$

with the generic $s$-variate coefficient $b_{S}(s), s=0,1, \cdots, N$, for the $S$-variate approximation yet to be determined. Of $N+1$ such coefficients, the last one,

$$
b_{S}(N)=\sum_{k=0}^{S}\left(\begin{array}{c}
N-S+k-1 \\
k
\end{array}\right)^{2}\left(\begin{array}{c}
N \\
S-k
\end{array}\right),
$$

is easier to determine. It is obtained from the expansion coefficients of the square in (4.24) that are associated with all variance terms of $\sigma^{2}$. To determine other coefficients, $b_{S}(s), s=0,1, \cdots, N-1$, the procedure used before for the univariate or bivariate approximation is unwieldy. An alternative scheme proposed here stems from the realization that the expressions of those coefficients consist of terms from two sources: (1) terms that depend solely on $N$, which can be described by a function $f$, say, of $N$; and (2) terms that depend on both $N$ and $s$, which can be described by another function $g$, say, of $N$ and $s$. Following this rationale, let a generic coefficient be expressed by

$$
b_{S}(s)=f(N)-g(N, s)
$$

for any $0 \leq N<\infty$ and $0 \leq s \leq N$. Switching the variables $N$ and $s$, (4.26) produces

$$
b_{S}(N)=f(s)-g(s, N) .
$$

At $s=N$, (4.26) and (4.27) result in $g(s, s)=g(N, N)=0$. Either of these two equations at $s=N$ with $g(s, s)=g(N, N)=0$ in mind yields $f(N)=b_{S}(N)$ or $f(s)=b_{S}(s)$, where the function $b_{S}$ is already described in (4.25). Therefore, the generic $s$-variate coefficient

$$
b_{S}(s)=\sum_{k=0}^{S}\left(\begin{array}{c}
s-S+k-1 \\
k
\end{array}\right)^{2}\left(\begin{array}{c}
s \\
S-k
\end{array}\right), 0 \leq s \leq N,
$$


where the binomial coefficients should be interpreted more generally than their classical combinatorial definition; for instance,

$$
\left(\begin{array}{l}
r \\
k
\end{array}\right):= \begin{cases}\frac{1}{k !} r^{-\underline{k}}=\frac{1}{k !} r(r-1) \cdots(r-k+1) & \text { if } k>0, \\
1 & \text { if } k=0, \\
0 & \text { if } k<0,\end{cases}
$$

valid for any real number $r \in \mathbb{R}$ and any integer $k \in \mathbb{Z}$. Adding all terms in (4.22), (4.23), and (4.24), with the cognizance that $b_{S}(s)=1$ for all $0 \leq s \leq S$, yields (4.19), proving the theorem.

Corollary 4.13. The lower and upper bounds of the expected error $\mathbb{E}\left[e_{S, R}\right]$ from the $S$-variate $R D D$ approximation, expressed in terms of the error $e_{S, A}$ from the $S$-variate $A D D$ approximations, are

$$
2^{S+1} e_{S, A} \leq \mathbb{E}\left[e_{S, R}\right] \leq\left[1+\sum_{k=0}^{S}\left(\begin{array}{c}
N-S+k-1 \\
k
\end{array}\right)^{2}\left(\begin{array}{c}
N \\
S-k
\end{array}\right)\right] e_{S, A},
$$

$0 \leq S<N<\infty$, where the coefficients of the lower and upper bounds are obtained from

$$
1+b_{S}(S+1)=1+\sum_{k=0}^{S}\left(\begin{array}{c}
S+1 \\
S-k
\end{array}\right)=\sum_{k=0}^{S+1}\left(\begin{array}{c}
S+1 \\
k
\end{array}\right)=2^{S+1}
$$

and

$$
1+b_{S}(N)=1+\sum_{k=0}^{S}\left(\begin{array}{c}
N-S+k-1 \\
k
\end{array}\right)^{2}\left(\begin{array}{c}
N \\
S-k
\end{array}\right)
$$

respectively.

Remark 4.14. Both Theorem 4.12 and Corollary 4.13 are new and provide a general result pertaining to RDD error analysis for an arbitrary truncation. The specific results of the zero-variate or univariate or bivariate RDD approximation, derived in the preceding subsection, can be recovered by setting $S=0$ or 1 or 2 in 4.19) through (4.32). From Corollary 4.13, the expected error from the $S$-variate RDD approximation of a multivariate function is at least $2^{S+1}$ times larger than the error from the $S$-variate ADD approximation. In other words, the ratio of RDD to ADD errors doubles for each increment of the truncation. Consequently, ADD approximations are exceedingly more precise than RDD approximations at highervariate truncations.

Although the relative disadvantage of using RDD over ADD worsens drastically with the truncation $S$, one hopes that the approximation error is also decreasing with increasing $S$. For instance, given a rate at which $\sigma_{u}^{2}$ decreases with $|u|$, what can be inferred from how fast $e_{S, A}$ and $\mathbb{E}\left[e_{S, R}\right]$ decay with respect to $S$ ? Corollary 4.15 and subsequent discussions provide some insights.

Corollary 4.15. If the variance of the zero-mean $A D D$ component function $y_{u, A}$ diminishes according to $\sigma_{u}^{2} \leq C p^{-|u|}$, where $\emptyset \neq u \subseteq\{1, \cdots, N\}$, and $C>0$ and $p>1$ are two real-valued constants, then

$$
e_{S, A} \leq C \sum_{s=S+1}^{N}\left(\begin{array}{c}
N \\
s
\end{array}\right) p^{-s}
$$


and

$$
\mathbb{E}\left[e_{S, R}\right] \leq C \sum_{s=S+1}^{N}\left[1+\sum_{k=0}^{S}\left(\begin{array}{c}
s-S+k-1 \\
k
\end{array}\right)^{2}\left(\begin{array}{c}
s \\
S-k
\end{array}\right)\right]\left(\begin{array}{c}
N \\
s
\end{array}\right) p^{-s}
$$

for $0 \leq S<N<\infty$.

When the equality holds in (4.33), $e_{S, A}$ decays strictly monotonically with respect to $S$ for any rate parameter $p$. In contrast, $\mathbb{E}\left[e_{S, R}\right]$, according to (4.34), does not follow suit for an arbitrary $p$. However, there exists a minimum threshold, say, $p_{\text {min }}$, when crossed, $\mathbb{E}\left[e_{S, R}\right]$ also decays monotonically. The threshold can be determined from the condition that $\mathbb{E}\left[e_{0, R}\right]=\mathbb{E}\left[e_{1, R}\right]$, resulting in the relationship

$$
\frac{2}{p_{\min }}=\frac{(N-1)\left(1+\frac{1}{p_{\min }}\right)^{N}}{\left(1+p_{\min }\right)^{2}}
$$

between $N$ and $p_{\min }$. Equation (4.35) supports an exact solution of

$$
N=1+\frac{1}{\ln \left(1+\frac{1}{p_{\min }}\right)} W\left(2\left(1+p_{\min }\right) \ln \left(1+\frac{1}{p_{\min }}\right)\right)
$$

in terms of $p_{\min }$, expressed by employing the Lambert $\mathrm{W}$ function $W$, and can be inverted easily. For instance, when $N=20$, (4.36) yields $p_{\min }=21.5187$, the only real-valued solution of interest. Depicted in Figure1 (left), $p_{\min }$ derived from (4.36) increases monotonically and strikingly close to linearly with $N$ for the ranges of the variables examined.

Using the equalities in (4.33) and (4.34), Figure 1 (right) presents plots of two normalized errors, $\mathbb{E}\left[e_{S, R}\right] / \sigma^{2}$ and $e_{S, A} / \sigma^{2}$, against $S$, each obtained for $N=20$ and $p=5$ or 50 , where the variance $\sigma^{2}=C\left[\left(1+\frac{1}{p}\right)^{N}-1\right]$. When the rate parameter is sufficiently low (e.g., $p=5<p_{\min }$ ), the expected RDD error initially rises before falling as $S$ becomes larger. The non-monotonic behavior of the RDD error is undesirable, but it vanishes when the rate parameter is sufficiently high (e.g., $p=50>p_{\min }$ ). No such anomaly is found in the ADD error for any $p$.

Corollary 4.16. The expected error $\mathbb{E}\left[e_{N-1, R}\right]$ from the best $R D D$ approximation, expressed in terms of the error $e_{N-1, A}$ from the best ADD approximation, where the best approximations are obtained by setting $S=N-1$, is

$$
\mathbb{E}\left[e_{N-1, R}\right]=2^{N} e_{N-1, A}, 1 \leq N<\infty .
$$

Remark 4.17. Due to the factor $2^{N}$ in (4.37), the expected error from the best RDD approximation as $N \rightarrow \infty$ can be significantly large unless the best ADD approximation commits an error equal to or smaller than $2^{-N}$. In reference to Corollary 4.15, suppose that $\sigma_{u}^{2} \leq C p^{-|u|}$. Then $\mathbb{E}\left[e_{N-1, R}\right] \leq C(2 / p)^{N}$. Therefore, $\mathbb{E}\left[e_{N-1, R}\right] \rightarrow 0$ as $N \rightarrow \infty$ for $p>2$.

The error analysis presented in this paper pertains to only second-moment characteristics of $y(\mathbf{X})$. Similar analyses or definitions aimed at higher-order moments or probability distribution of $y$ can be envisioned, but no closed-form solutions and simple expressions are possible. However, if $y$ satisfies the requirements of the Chebyshev inequality or its descendants - a condition fulfilled by many realistic 

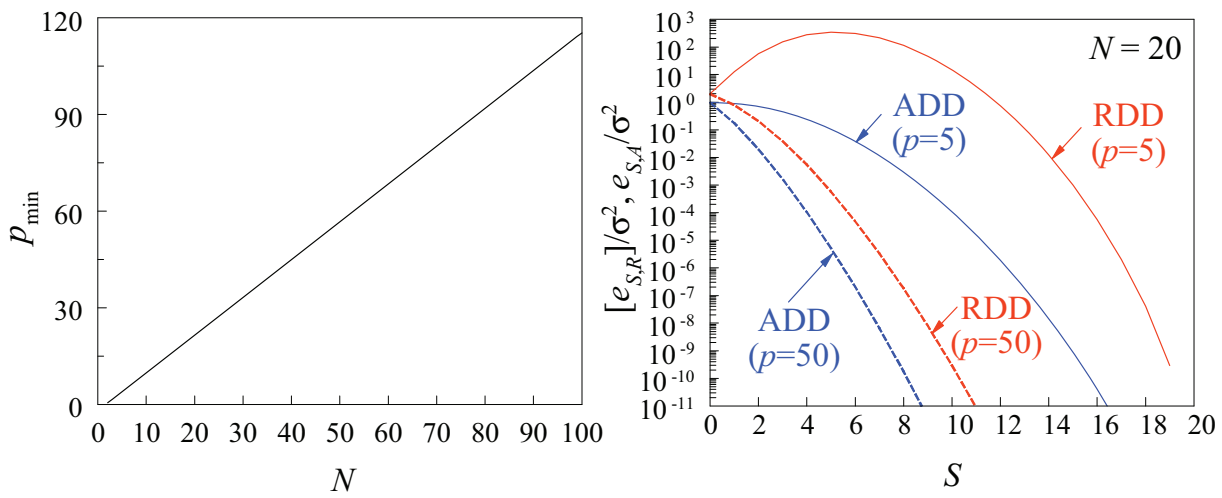

FIGURE 1. Relationship between $p_{\min }$ and $N$ (left) and normalized $\mathrm{RDD}$ and ADD errors versus $S$ for $N=20, p=5$ or 50 (right).

functions - then the results and findings from this work can be effectively exploited for stochastic analysis. See the longer version of the paper for further details.

\section{Conclusions}

Two variants of dimensional decomposition, namely, RDD and ADD, of a multivariate function, both representing finite sums of lower-dimensional component functions, were studied. The approximations resulting from the truncated RDD and ADD were explicated, including clarifications of parallel developments and synonyms used by various researchers. For the RDD approximation, a direct form, previously developed by the author's group, was found to provide a vital link to subsequent error analysis. New theorems were proven about the expected errors from the bivariate and general RDD approximations, so far available only for the univariate RDD approximation, when the reference point is selected randomly. They furnish new formulae for the lower and upper bounds of the expected error committed by an arbitrarily truncated RDD, providing a means to grade RDD against ADD approximations. The formulae indicate that the expected error from the $S$ variate RDD approximation of a function of $N$ variables, where $0 \leq S<N<\infty$, is at least $2^{S+1}$ times larger than the error from the $S$-variate ADD approximation. Consequently, ADD approximations are exceedingly more precise than RDD approximations at higher-variate truncations. The analysis also finds the RDD approximation to be sub-optimal for an arbitrarily selected reference point, whereas the ADD approximation always results in minimum error. Therefore, RDD approximations should be used with caveat.

\section{REFERENCES}

[1] Wassily Hoeffding, A class of statistics with asymptotically normal distribution, Ann. Math. Statistics 19 (1948), 293-325. MR0026294(10,134g)

[2] Art B. Owen, The dimension distribution and quadrature test functions, Statist. Sinica 13 (2003), no. 1, 1-17. MR.1963917 (2004a:65006)

[3] Il'ya M. Sobol', Mnogomernye Kvadraturnye Formuly i Funktsii Khaara (Russian), Izdat. "Nauka", Moscow, 1969. MR0422968 (54 \#10952) 
[4] Il'ya M. Sobol', Theorems and examples on high dimensional model representations, Reliab. Eng. Syst. Safe. 79 (2003), 187-193.

[5] Bradley Efron and Charles Stein, The jackknife estimate of variance, Ann. Statist. 9 (1981), no. 3, 586-596. MR615434 (82k:62074)

[6] Art B. Owen, Monte Carlo variance of scrambled net quadrature, SIAM J. Numer. Anal. 34 (1997), no. 5, 1884-1910, DOI 10.1137/S0036142994277468. MR1472202(98h:65006)

[7] Fred J. Hickernell, Quadrature error bounds with applications to lattice rules, SIAM J. Numer. Anal. 33 (1996), no. 5, 1995-2016, DOI 10.1137/S0036142994261439. MR1411860 (97m:65050)

[8] Herschel Rabitz and Ömer F. Alış, General foundations of high-dimensional model representations, J. Math. Chem. 25 (1999), no. 2-3, 197-233, DOI 10.1023/A:1019188517934. MR.1731292

[9] Sharif Rahman and Heqin Xu, A univariate dimension-reduction method for multidimensional integration in stochastic mechanics, Probabilist. Eng. Mech. 19 (2004), 393-408.

[10] Hequin $\mathrm{Xu}$ and Sharif Rahman, A generalized dimension-reduction method for multidimensional integration in stochastic mechanics, Internat. J. Numer. Methods Engrg. 61 (2004), 1992-2019.

[11] Hequin $\mathrm{Xu}$ and Sharif Rahman, Decomposition methods for structural reliability analysis, Probabilist. Eng. Mech. 20 (2005), 239-250.

[12] Russell E. Caflisch, William Morokoff, and Art Owen, Valuation of mortgage backed securities using Brownian bridges to reduce effective dimension, J. Comput. Finance 1 (1997), 27-46.

[13] Xiaoqun Wang, On the approximation error in high dimensional model representation, In Proceedings of 40th Conference on Winter Simulation, 453-462, 2008.

[14] Frances Y. Kuo, Ian H. Sloan, Grzegorz W. Wasilkowski, and Henryk Woźniakowski, On decompositions of multivariate functions, Math. Comp. 79 (2010), no. 270, 953-966, DOI 10.1090/S0025-5718-09-02319-9. MR2600550 (2011a:41039)

[15] Sharif Rahman, A polynomial dimensional decomposition for stochastic computing, Internat. J. Numer. Methods Engrg. 76 (2008), no. 13, 2091-2116, DOI 10.1002/nme.2394. MR2475282 (2009k:65008)

[16] Sharif Rahman, Extended polynomial dimensional decomposition for arbitrary probability distributions, J. Eng. Mech-ASCE 135 (2009), 1439-51.

[17] Fred J. Hickernell, Ian H. Sloan, and Grzegorz W. Wasilkowski, On tractability of weighted integration over bounded and unbounded regions in $\mathbb{R}^{s}$, Math. Comp. 73 (2004), no. 248, 1885-1901 (electronic), DOI 10.1090/S0025-5718-04-01624-2. MR2059741 (2005c:65018)

[18] Michael Griebel and Markus Holtz, Dimension-wise integration of high-dimensional functions with applications to finance, J. Complexity 26 (2010), no. 5, 455-489, DOI 10.1016/j.jco.2010.06.001. MR2719643(2011i:65038)

[19] Il'ya M. Sobol', Global sensitivity indices for nonlinear mathematical models and their Monte Carlo estimates, Math. Comput. Simulation 55 (2001), no. 1-3, 271-280, DOI 10.1016/S03784754(00)00270-6. The Second IMACS Seminar on Monte Carlo Methods (Varna, 1999). MR.1823119

[20] Sharif Rahman, Decomposition methods for structural reliability analysis revisited, Probabilist. Eng. Mech. 26 (2011), 357-363.

Applied Mathematical and Computational Sciences, The University of Iowa, Iowa CiTy, IowA 52242

E-mail address: sharif-rahman@uiowa.edu 